\title{
Short communication: Rapid detection of milk fat adulteration with vegetable oil by fluorescence spectroscopy
}

\author{
M. P. Ntakatsane, ${ }^{*} \dagger$ X. M. Liu, ${ }^{*}$ and P. Zhou* ${ }^{* 1}$ \\ *State Key Laboratory of Food Science and Technology, School of Food Science and Technology, Jiangnan University, \\ Wuxi, Jiangsu Province 214122, China \\ †Department of Animal Science, Faculty of Agriculture, National University of Lesotho, Roma 180, Lesotho, Southern Africa
}

\begin{abstract}
This study assessed the potential application of fluorescence spectroscopy in detecting adulteration of milk fat with vegetable oil and characterizing the samples according to the source of the fat. Pure butterfat was adulterated with different vegetable oils at various concentrations $(0,5,10,15,20,30$, and $40 \%)$. Nonfat and reduced-fat milk were also adulterated with vegetable oils to simulate full-fat milk (3.2\%). The 2 - and 3 -dimensional front-face fluorescence spectroscopy and gas chromatography were used to obtain the fluorescence spectra and fatty acid profile, respectively. Principal component analysis and 3-way partial least squares regression analysis were applied to analyze the data. The pure and adulterated samples were discriminated based on the total concentration of saturated fatty acids and unsaturated fatty acids, and also on the 3 major fluorophores: tryptophan, tocopherols, and riboflavin. Fluorescence spectroscopy was able to detect up to $5 \%$ of adulteration of vegetable oil into the butterfat. The saturated fatty acids showed higher predictability than the unsaturated fatty acids $\left(\mathrm{R}^{2}=0.73-0.92\right.$ vs. $0.20-0.65$, respectively). The study demonstrated the high potential of fluorescence spectroscopy to rapidly detect adulteration of milk fat with vegetable oil, and discriminate commercial butter and milk according to the source of the fat.
\end{abstract}

Key words: detection, milk fat adulteration, vegetable oil, fluorescence spectroscopy

\section{Short Communication}

Milk fat is an important food component and plays a significant role in the economics, nutrition, and physical and chemical properties of milk and milk-derived products; it is also a good source of fat-soluble vitamins and essential FA (Kumar et al., 2010). Due to

Received November 26, 2012.

Accepted December 28, 2012

${ }^{1}$ Corresponding author: zhoupeng@jiangnan.edu.cn increased demand, milk fat has been a target of the fraudulent practices, such as replacement with cheaper or more readily available non-milk fats of plant and animal origin. Replacement of milk fat with fat from other sources is not only an economic fraud, but may also pose a risk to human health.

Fatty acid composition of milk fat has long been used as criteria to detect adulteration with vegetable oil, mainly because milk fat is characteristic of shortchain FA (Molkentin and Precht, 1998), whereas vegetable oils have medium- to long-chain FA. However, FA composition is influenced by several factors, such as nutrition and genetics. Several methods have been developed to date for detection of milk fat adulteration, some of which are laborious and expensive, thereby limiting the application on a regular basis. Conversely, spectroscopic methods yield information on the components of a mixture in one spectrum, and usually without the need for derivatization (Knothe and Kenar, 2004). Although fluorescence spectroscopy has been applied in several studies, the use of multidimensional fluorescence spectroscopy in dairy products has not been widely explored, and very limited information is available in this area. Therefore, the objective of the study was to evaluate the potential application of 2 - and 3-dimensional (3D) front-face fluorescence spectroscopy, combined with chemometric tools, to detect adulteration of vegetable oil in milk fat and characterize the samples according to the source of adulterant oil and the level of adulteration.

Commercial butter, full-fat $(3.2 \%)$, reduced-fat $(1.2 \%)$, and nonfat milks, as well as vegetable oils (sunflower, canola, maize, and rice bran) were obtained from the local supermarket. The reagents (methanol, hexane, sodium hydroxide, boron triflouride, sodium chloride, and anhydrous sodium acetate) were obtained from Sigma-Aldrich (Shanghai, China). First, the butter was melted at $60^{\circ} \mathrm{C}$, centrifuged at $11,180 \times g$ for $10 \mathrm{~min}$ at $20^{\circ} \mathrm{C}$, and filtered through a filter paper. The filtered butterfat was adulterated with canola and sunflower oils at various concentrations $(0,5,10,15$, 20,30, and 40\%). The butterfat was also adulterated 
with rice bran and maize oils for comparison purposes. The adulterated butterfat samples were prepared in 2 groups; one was used for gas chromatographic analysis, whereas the other was used for fluorescence measurement (dissolved in n-hexane before measurement). Next, the 2 types of adulterated milks were prepared to simulate full-fat milk (3.2\%), and the samples consisted of the complete replacement and partial supplementation of milk fat with vegetable oil. The samples were homogenized (Nano homogenize machine, AH basic model, ATS Engineering Inc., Shanghai, China) to ensure thorough mixing of milk and oil. The commercial milk from different manufacturers were obtained and used as control. For FA analysis, milk fat separation was carried out following the rapid method developed by Feng et al., (2004) with minor modifications. Briefly, $30 \mathrm{~mL}$ of milk samples were tempered to $20^{\circ} \mathrm{C}$ and centrifuged (Multifuge X1R Centrifuge, Heraeus, Trenton, $\mathrm{NJ})$ at $12,555 \times g$ for $30 \mathrm{~min}$. The fat layer was transferred to micro tubes and allowed to stand for $30 \mathrm{~min}$ at room temperature before being centrifuged at 14,737 $\times g$ for 20 min at the same temperature. The top layer was taken for analysis.

The milk fat and vegetable oil mixtures were converted to FA methyl esters using sodium hydroxide. A portion of $0.10 \mathrm{~g}$ butterfat or vegetable oil was added into test tubes with caps. Two milliliters of $0.5 \mathrm{~mol} / \mathrm{L}$ $\mathrm{NaOH}$ (in methanol) was added to the mixture, tightly capped, and placed in a water bath for $30 \mathrm{~min}$ at $60^{\circ} \mathrm{C}$. Two milliliters of $25 \%$ boron trifluoride solution (in methanol) was added to the mixture and kept in a water-bath for $20 \mathrm{~min}$ at $60^{\circ} \mathrm{C}$. After cooling to room temperature, $2 \mathrm{~mL}$ of $\mathrm{n}$-hexane was added and stirred. Two milliliters of saturated $\mathrm{NaCl}$ solution was added, and the top organic part was transferred into a dry test tube after centrifugation for $10 \mathrm{~min}$ at $1,789 \times \mathrm{g}$. Anhydrous sodium acetate was added to the solution to remove the residual water, and the top layer of the solution was transferred into the sample tubes for subsequent chromatographic analysis. The samples were prepared in quadruplicates and 2 replicates were mixed together to form duplicate samples used for analysis.

Gas chromatography was used to determine the FA composition of the samples. Analysis was carried out on Shimadzu GC-2010 gas chromatograph (Kyoto, Japan) equipped with flame ionization detector and a CP-WAX column $(30 \mathrm{~m} \times 0.32 \mathrm{~mm} ; 0.25 \mu \mathrm{m}$ film thickness $)$. The injector and detector temperatures were both set at $250^{\circ} \mathrm{C}$. The carrier gas was nitrogen at the column flow rate of $3 \mathrm{~mL} / \mathrm{min}$, the fuel gas was hydrogen at the flow rate of $47 \mathrm{~mL} / \mathrm{min}$, and the oxidant gas was air at the flow rate of $400 \mathrm{~mL} / \mathrm{min}$. To optimize the conditions, the column temperature was programmed as follows: $120^{\circ} \mathrm{C}$ in the beginning for $3 \mathrm{~min}$, increased to $190^{\circ} \mathrm{C}$ 
Table 2. Unsaturated fatty acid composition (\%) of commercial and adulterated milks

\begin{tabular}{|c|c|c|c|c|c|}
\hline Sample $^{1}$ & $\begin{array}{l}\text { Palmitoleic } \\
\text { C16:1 }\end{array}$ & $\begin{array}{l}\text { Oleic } \\
\text { C18:1 }\end{array}$ & $\begin{array}{c}\text { Linoleic } \\
\text { C18:2 }\end{array}$ & $\begin{array}{c}\text { Linolenic } \\
\text { C18:3 }\end{array}$ & $\begin{array}{c}\text { Gadoleic } \\
\text { C20:1 }\end{array}$ \\
\hline CW1 & $1.43 \pm 0.03^{\mathrm{e}}$ & $26.19 \pm 0.04^{\mathrm{b}}$ & $3.20 \pm 0.03^{\mathrm{b}}$ & $0.37 \pm 0.03^{\mathrm{bc}}$ & $0.13 \pm 0.01^{\mathrm{b}}$ \\
\hline CW2 & $1.34 \pm 0.03^{\mathrm{de}}$ & $25.67 \pm 0.04^{\mathrm{a}}$ & $4.04 \pm 0.03^{\mathrm{d}}$ & $0.43 \pm 0.03^{\mathrm{c}}$ & $0.15 \pm 0.01^{\mathrm{a}}$ \\
\hline CW3 & $1.27 \pm 0.03^{\mathrm{d}}$ & $29.13 \pm 0.03^{\mathrm{e}}$ & $3.71 \pm 0.03^{c}$ & $0.22 \pm 0.03^{\mathrm{a}}$ & $\mathrm{ND}^{2}$ \\
\hline CW4 & $1.42 \pm 0.03^{\mathrm{e}}$ & $26.89 \pm 0.03^{\mathrm{c}}$ & $3.05 \pm 0.03^{\mathrm{a}}$ & $0.39 \pm 0.03^{\mathrm{bc}}$ & ND \\
\hline $\mathrm{SFc}$ & $0.94 \pm 0.02^{\mathrm{bc}}$ & $39.46 \pm 0.04^{\mathrm{i}}$ & $9.71 \pm 0.02^{\mathrm{e}}$ & $2.84 \pm 0.02^{\mathrm{h}}$ & $0.52 \pm 0.03^{\mathrm{c}}$ \\
\hline SFf & $0.92 \pm 0.02^{\mathrm{bc}}$ & $27.64 \pm 0.03^{\mathrm{d}}$ & $23.76 \pm 0.03^{\mathrm{i}}$ & $0.36 \pm 0.03^{\mathrm{bc}}$ & ND \\
\hline SFm & $1.02 \pm 0.02^{\mathrm{c}}$ & $29.81 \pm 0.03^{\mathrm{f}}$ & $16.85 \pm 0.02^{\mathrm{f}}$ & $0.56 \pm 0.03^{\mathrm{d}}$ & ND \\
\hline $\mathrm{SFr}$ & $0.84 \pm 0.02^{\mathrm{b}}$ & $33.39 \pm 0.03^{\mathrm{h}}$ & $18.98 \pm 0.03^{\mathrm{g}}$ & $0.71 \pm 0.01^{\mathrm{e}}$ & $0.23 \pm 0.01^{\mathrm{b}}$ \\
\hline $\mathrm{RFc}$ & $0.28 \pm 0.03^{\mathrm{a}}$ & $59.01 \pm 0.04^{\mathrm{k}}$ & $19.53 \pm 0.03^{\mathrm{h}}$ & $6.91 \pm 0.03^{\mathrm{i}}$ & $1.31 \pm 0.02^{\mathrm{d}}$ \\
\hline RFf & $0.17 \pm 0.01^{\mathrm{a}}$ & $25.69 \pm 0.03^{\mathrm{a}}$ & $58.29 \pm 0.04^{1}$ & $0.28 \pm 0.01^{\mathrm{ab}}$ & $0.16 \pm 0.01^{\mathrm{b}}$ \\
\hline RFm & $0.22 \pm 0.01^{\mathrm{a}}$ & $31.36 \pm 0.03^{\mathrm{g}}$ & $49.28 \pm 0.04^{\mathrm{k}}$ & $0.93 \pm 0.01^{\mathrm{f}}$ & $0.18 \pm 0.01^{\mathrm{b}}$ \\
\hline $\mathrm{RFr}$ & $0.26 \pm 0.04^{\mathrm{a}}$ & $39.69 \pm 0.04^{\mathrm{j}}$ & $35.86 \pm 0.04^{\mathrm{j}}$ & $1.00 \pm 0.02^{\mathrm{g}}$ & $0.43 \pm 0.01^{\mathrm{c}}$ \\
\hline
\end{tabular}

${ }^{\mathrm{a}-\mathrm{l}}$ Values within a column with different letters are different $(P<0.05)$.

${ }^{1}$ The uppercase letters in the sample names represent the type of milk $(\mathrm{CW}=$ commercial whole milk; $\mathrm{RF}=$ replaced fat; $\mathrm{SF}=$ supplemented fat), whereas the numbers and the lowercase letters represent the various manufacturers and adulterant oils, respectively $(1-4=$ manufacturers; $\mathrm{c}=$ canola; $\mathrm{f}=$ sunflower; $\mathrm{m}=$ maize; $\mathrm{r}=$ rice bran).

${ }^{2} \mathrm{ND}=$ not detected.

at the rate of $10^{\circ} \mathrm{C} / \mathrm{min}$, increased further to $220^{\circ} \mathrm{C}$ at the rate of $2^{\circ} \mathrm{C} / \mathrm{min}$, and then maintained for $15 \mathrm{~min}$. A sample of $0.8 \mu \mathrm{L}$ was injected with the split ratio of 1:8. Fatty acids were identified by comparing their retention times with those of the authentic samples kept in a library of references within the system, as well as data from literature. The results were expressed as percentage by weight $(\%, \mathrm{wt} / \mathrm{wt})$ of the total $\mathrm{FA}$; the samples were analyzed in duplicates.

Fluorescence spectra were obtained from F-7000 FL spectrophotometer (Hitachi High Technology, Tokyo, Japan) with the incidence angle of excitation at $56^{\circ}$, with the excitation and emission slits both set at $5 \mathrm{~nm}$. The excitation spectra of vitamin A (250-390 nm) were recorded on the samples with emission wavelength set at $410 \mathrm{~nm}$. The 3D front-face fluorescence scan was performed with emission at 310 to $700 \mathrm{~nm}$ (interval of $5 \mathrm{~nm}$ ) and excitation at 250 to $550 \mathrm{~nm}$ (interval of 10 $\mathrm{nm})$. The samples were analyzed in duplicates with 3 scans performed on each replicate.

Principal component analysis (PCA) was applied to the fatty acid profile and the fluorescence spectral data separately. The $t$-test (paired) was used for the comparison of the principal component scores $(P<0.05)$ between the pure and adulterated butterfat samples. Corrected 3D spectra were concatenated into excitationemission matrix before the 3-way partial least squares regression analysis was applied to decompose the data. The outliers were removed from the data. The statistical analysis was carried out using the Unscrambler v9.7 (Camo Software, Domlur, India) and SPSS 17.0 (SPSS Inc., Chicago, IL). Tukey's test was used at $P<0.05$ to determine the level of significance of the differences between the sample means.
The FA profile of the samples is shown in Tables 1 and 2. The commercial milk fat was rich in shortto medium-chain SFA, whereas the oil-adulterated samples displayed high concentration of long-chain unsaturated fatty acids (UFA). Adulteration of reducedfat and nonfat milks with vegetable oil decreased the content of short-chain FA and SFA (except arachidic acid, C20:0), but the opposite was true for the UFA content. Kennelly (1996) indicated that milk fat contains significantly higher concentrations of short- and medium-chain FA and relatively lower concentrations of UFA, as compared with other dietary sources of vegetable and animal fat. The PCA revealed that the pure and adulterated samples were discriminated according to the total concentration of SFA and UFA respectively (data not shown).

The normalized vitamin A spectra of the samples adulterated with canola and sunflower oils displayed similar patterns, with emission maxima at around 322 nm. Figure 1a shows the normalized spectra of butterfat adulterated with canola oil. Increasing the oil concentration resulted in slight decreases in fluorescence intensity with no significant changes in excitation maximum. Moreover, the continuous adulteration resulted in the gradual disappearance of the peak at $308 \mathrm{~nm}$, the typical peak for vitamin A spectra of milk fat. However, canola and sunflower oils seem to possess fluorescent properties similar to the butterfat, which made visual identification of adulteration from the spectra difficult without the application of chemometric tools. Conversely, the adulteration of butterfat with rice bran and maize oils resulted in dramatic changes in the spectra, which were characterized by a distinct, continuous decrease in fluorescence intensity and the 
a)

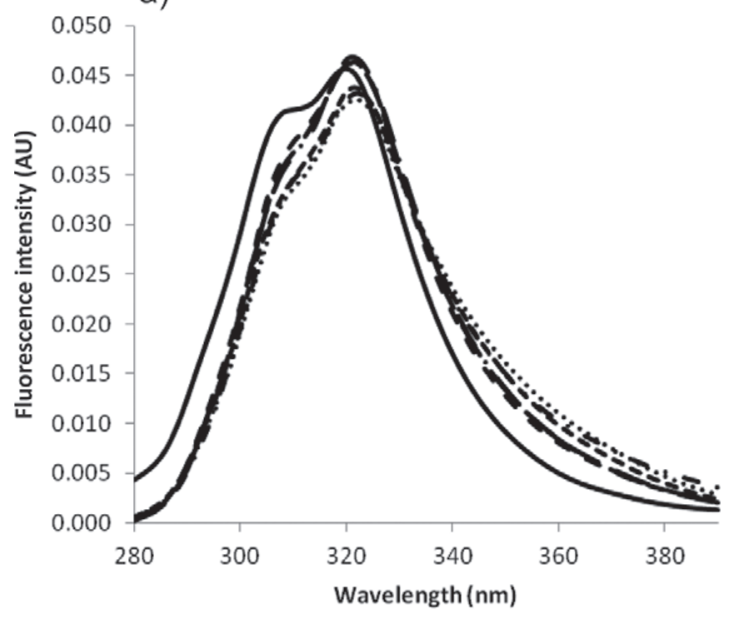

b)

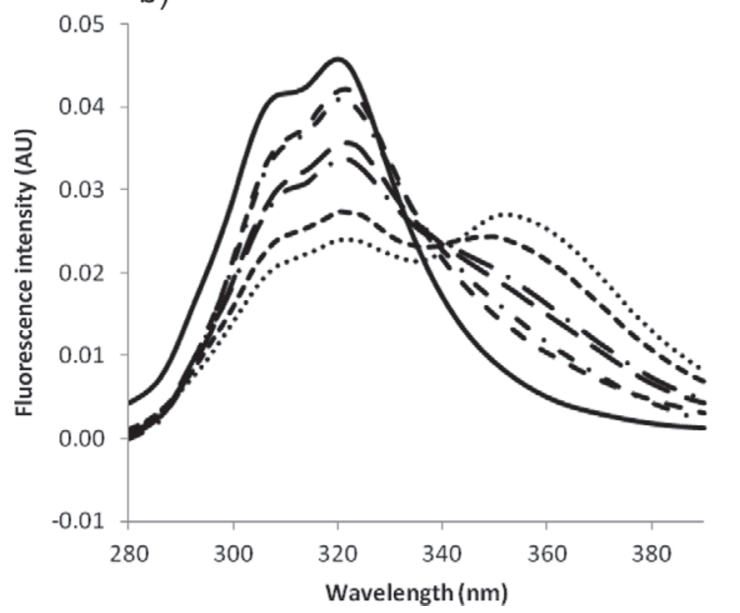

Figure 1. Vitamin A excitation spectra for pure butterfat and the butterfat adulterated with vegetable oils recorded after emission at $410 \mathrm{~nm}$. First and second letters in the sample names represent butter (B) and type of adulterant oil $(\mathrm{C}=$ canola; $\mathrm{R}=$ rice bran $)$, respectively, and the numbers represent the concentration (\%) of oil in the mixtures $(0,5,10,15,20,30,40)$.

development of new peak at a longer wavelength $(352$ $\mathrm{nm}$ ) as the concentration increased. The spectra of butterfat adulterated with rice bran are shown in Figure 1b. The variations in the spectral patterns might be related to, but not limited to, the source of the plant, time of harvest, stability after harvest, refining procedure, commercial hydrogenation procedures (Herting and Drury, 1963), or FA profiles.

Considering the PCA similarity map for the normalized vitamin A spectra of the pure and adulterated butterfat samples, the samples were discriminated according to the first 2 principal components (PC), which accounted for $99 \%$ of the total variance (Figure 2a). Principal component $1(70 \%)$ discriminated the samples according to the increasing oil concentration, a)

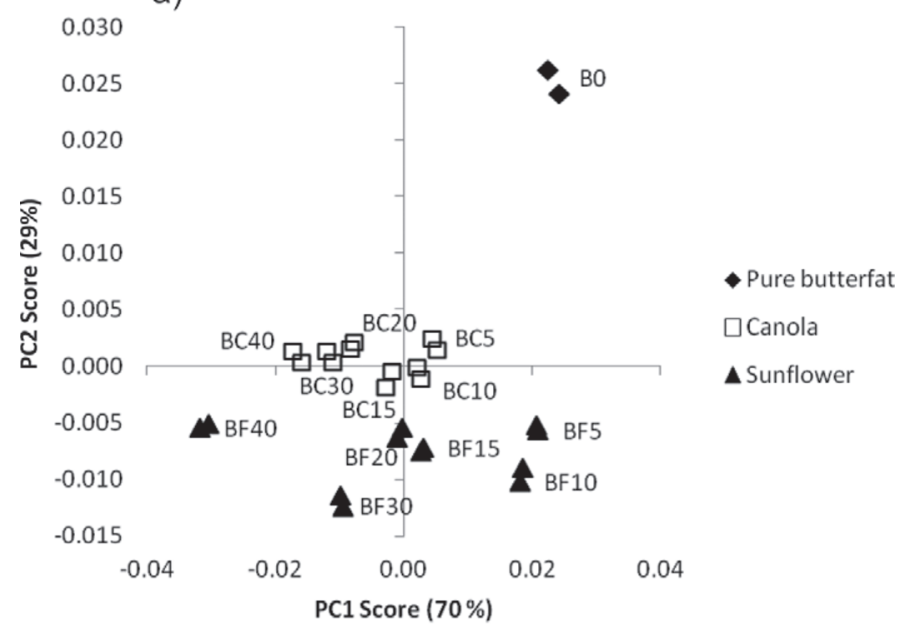

b)

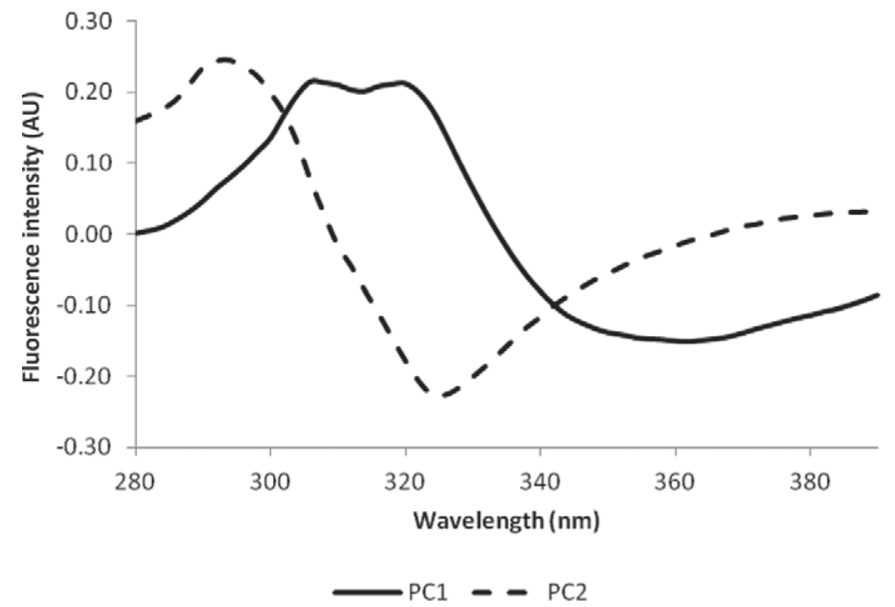

Figure 2. Principal component (PC) analysis similarity map (a) and factor loadings (b) for vitamin A excitation spectra of pure and oil-adulterated butterfat samples. The letters in the sample names represent butter $(\mathrm{B})$ and type of adulterant oil $(\mathrm{C}=$ canola; $\mathrm{F}=$ sunflower), respectively, and the numbers represent the concentration (\%) of oil in the mixtures $(0,5,10,15,20,30,40)$.

with scores decreasing as concentration increased. The comparison of PC1 scores for the pure and adulterated samples showed a significant difference $(P<0.05)$ between the pure butterfat and the butterfat adulterated with oil at various levels (data not shown). The pure butterfat was clearly discriminated from all the adulterated samples based on PC2 (29\%) with a positive score. The factor loadings corresponding to PC1 displayed an opposition between 2 positive peaks at 306 and $322 \mathrm{~nm}$ and a broad, negative band at around $360 \mathrm{~nm}$, which may be attributed to vitamin A content in the samples (Figure 2b). It was reported that the vitamin A spectra display excitation maxima at around $322 \mathrm{~nm}$ (Karoui et al., 2007), with a small shoulder appearing at 304 to $308 \mathrm{~nm}$ (Zaïdi et al., 2008). The 

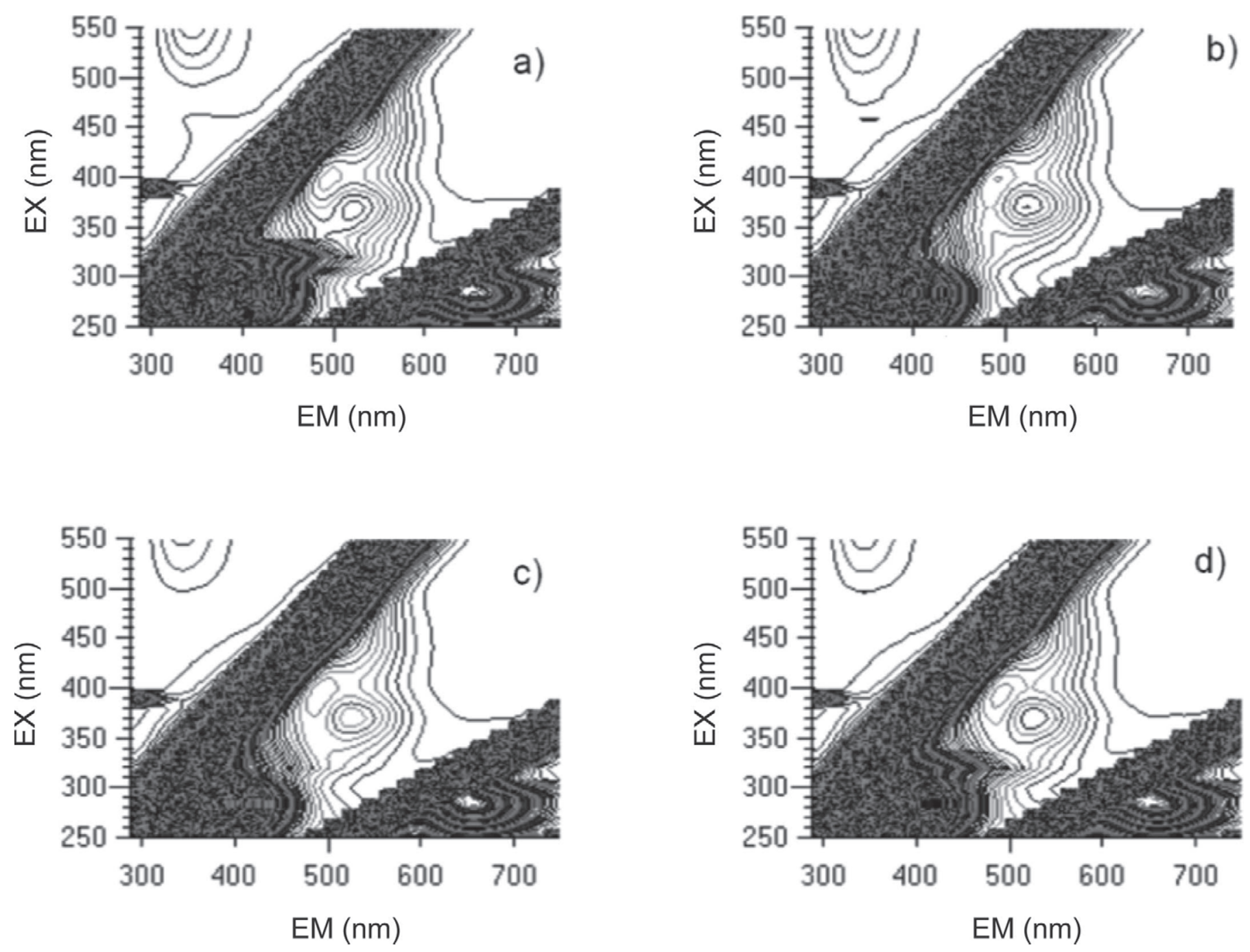

Figure 3. Contour maps for the three-dimensional fluorescence spectra of the pure commercial whole milk (a), nonfat milk (b), reduced fat milk adulterated with vegetable oil (c), and nonfat milk adulterated with oil (d). EX = excitation wavelength; EM = emission wavelength.

factor loadings corresponding to PC2 presented an opposition between a positive peak at $294 \mathrm{~nm}$ and a negative peak at $324 \mathrm{~nm}$, which may describe the variation in vitamin $\mathrm{A}$ content between the pure and oil-adulterated butterfat samples. According to the US Kiplinger magazine (Changing Times, 1952), butter contains vitamin $\mathrm{A}$ in varying amounts depending on the production season, whereas vegetable oils have no natural vitamin $\mathrm{A}$, which explains why products such as margarine are required by law to be fortified with vitamin $\mathrm{A}$. The negative peak at $324 \mathrm{~nm}$ may indicate a shift in the excitation maxima of the pure butterfat spectra due to the adulteration with vegetable oil.

The potential application of the 3D fluorescence spectroscopy to screen the milk system for the total fluorescence and discriminate between pure commercial milk and milk samples adulterated with vegetable oils was also evaluated. The contour maps of the $3 \mathrm{D}$ spectra displayed 3 prominent peaks, with an intense peak at excitation/emission of $280 / 345 \mathrm{~nm}$, a peak at 280/660 $\mathrm{nm}$, and another peak at 370/525 nm (Figure 3). The peaks were attributed to tryptophan in proteins, vitamin E compounds, and riboflavin, respectively, as indicated by Andersen and Mortensen (2008). Adulteration of milk with vegetable oil resulted in a decrease in fluorescence intensity with no changes in the excitation/ emission maxima except for the riboflavin, whereby a shift toward shorter emission wavelength was observed (525-515 nm).

The score plot from the 3-way partial least squares regression analysis displayed the discrimination of the samples according to the first 3 principal components. 
a)

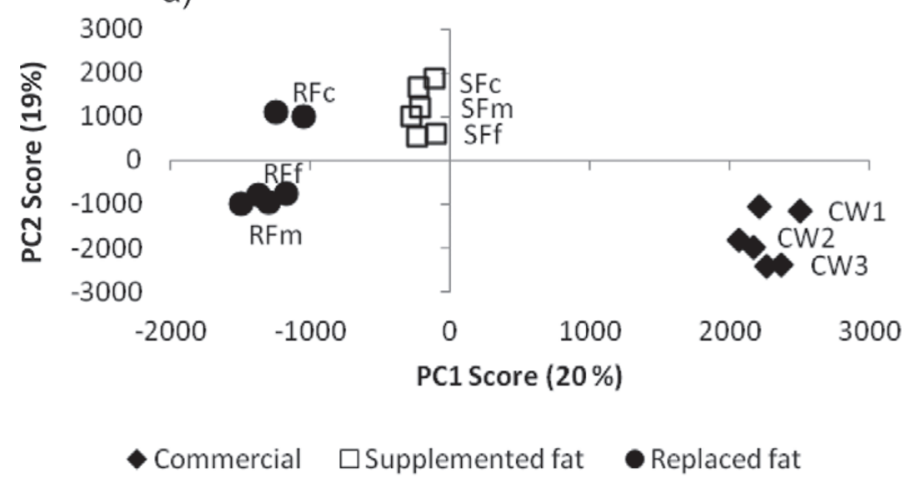

c)

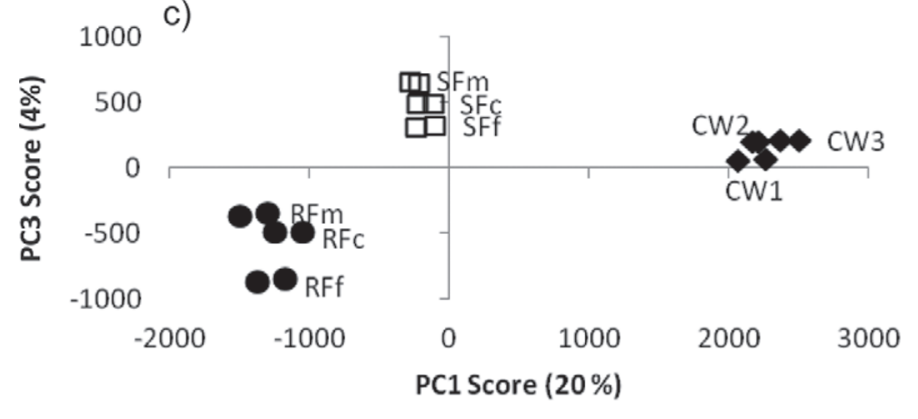

- Commercial asupplemented fat

- Replaced fat

e)

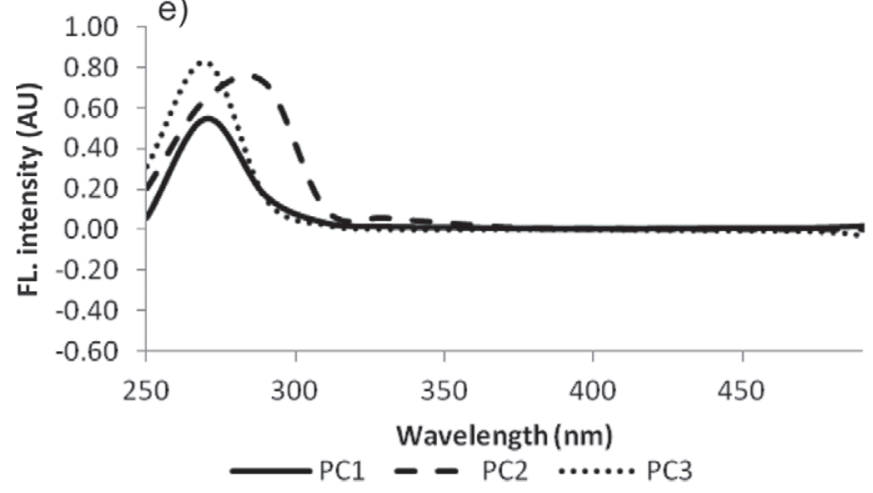

b)
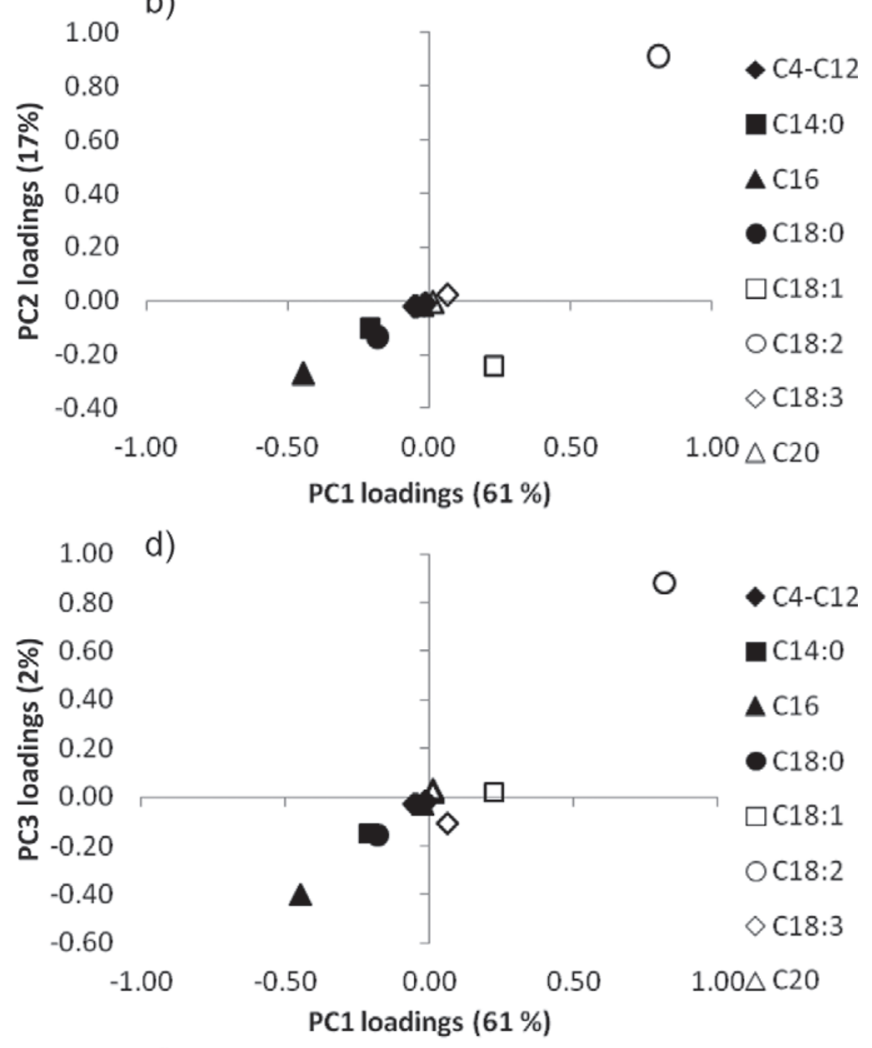

f)

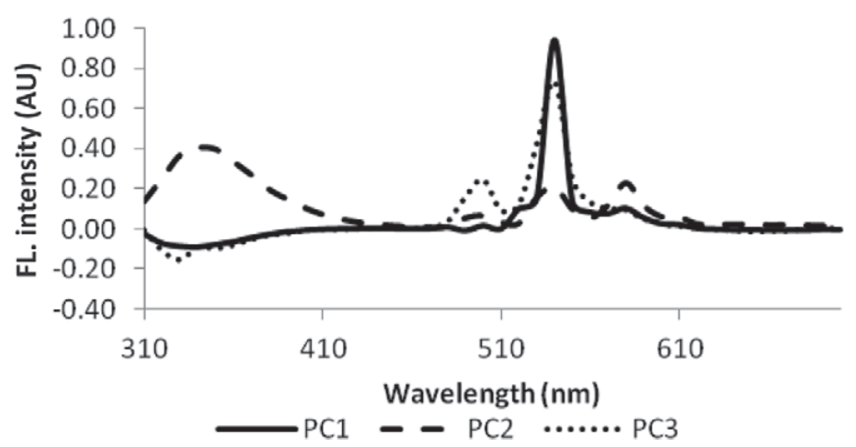

Figure 4. Three-way partial least squares regression analysis similarity maps (a and c; PC1 score), loadings corresponding to the fatty acid data (b and d; PC1 loadings), and fluorescence (FL) data (e and f; wavelength) for commercial and oil-adulterated milk samples. The uppercase letters in the sample names represent the type of milk $(\mathrm{CW}=$ commercial whole milk; $\mathrm{RF}=$ replaced fat; $\mathrm{SF}=$ supplemented fat $)$, while the numbers and lowercase letters represent the various manufacturers and adulterant oils $(1-4=$ manufacturers; $\mathrm{c}=\mathrm{canola} ; \mathrm{f}=\mathrm{sunflower} ; \mathrm{m}=$ maize). $\mathrm{PC}=$ principal component.

The 3 sample groups (commercial, replaced, and supplemented fat samples) were clearly separated according to PC1, as shown in Figure 4a. Two loading weights were considered in this case: the loadings corresponding to the fluorescence spectra and the FA data profile. The commercial and replaced fat samples were displayed on the extreme positive and negative sides of the plot, respectively, and this correlated well to SFA (palmitic acid mainly, myristic and stearic acids to some extent) and linoleic acid, respectively, as shown by the load- ings plot (Figure 4b). The canola-adulterated samples, which were characterized by high oleic acid content, were discriminated from others according to $\mathrm{PC} 2$ (19\%) with higher positive scores. The replaced fat samples were discriminated from others with negative PC3 scores (4\%) based on the high content of linoleic acid (Figure 4c and 4d).

Besides the matrix loadings corresponding to FA profile, the 3 principal components which discriminated the samples also corresponded to the fluorescent com- 
pounds in the samples (Figure 4e and 4f). The PC1 and PC2 displayed prominent peaks at excitation/emission of $270 / 530$ and $290 / 345 \mathrm{~nm}$, and these were ascribed to riboflavin and tryptophan, respectively. Riboflavin has been reported as a highly fluorescent compound with excitation maxima at 270,370, and $450 \mathrm{~nm}$ (Andersen and Mortensen, 2008). A small peak was also observed at around $325 / 490 \mathrm{~nm}$ on $\mathrm{PC} 2$, and this was attributed to vitamin $\mathrm{A}$ in the commercial samples. The $\mathrm{PC} 3$ showed 2 important peaks at excitation of $270 \mathrm{~nm}$ and emission of 330 and $530 \mathrm{~nm}$, and these were ascribed to the vitamin $\mathrm{E}$ and riboflavin, respectively.

Considering the measured versus predicted plot, it was observed that the established models were good for prediction of SFA, whereas the UFA was poorly correlated to the fluorescence data $\left(\mathrm{R}^{2}=0.73-0.91\right.$ vs. $0.20-0.65$ ). Oleic and linoleic acids displayed the lowest association $\left(\mathrm{R}^{2}<0.21\right)$. The study revealed the great potential of fluorescence spectroscopy to rapidly discriminate between authentic and adulterated milk fat, and also to classify the samples according to their source of fat. More importantly, fluorescence spectroscopy could detect up to $5 \%$ of vegetable oil in butterfat. However, further study is necessary to investigate the relationship between the fluorescence data and the SFA of fats and oils.

\section{REFERENCES}

Andersen, C. M., and G. Mortensen. 2008. Fluorescence spectroscopy: A rapid tool for analyzing dairy products. J. Agric. Food Chem. $56: 720-729$.

Changing Times. 1952. A report on what makes up the prices of various goods and services: Butter and margarine. April issue. The Kiplinger Magazine, Washington, DC.

Feng, S., A. L. Lock, and P. C. Garnsworthy. 2004. A rapid method for determining fatty acid composition of milk. J. Dairy Sci. 87:3785-3788.

Herting, D. C., and E. E. Drury. 1963. Vitamin E content of vegetable oils and fats. J. Nutr. 81:335-342.

Karoui, R., E. Dufour, and J. D. Baerdemaeker. 2007. Front face fluorescence spectroscopy coupled with chemometric tools for monitoring the oxidation of semi-hard cheeses throughout ripening. Food Chem. 101:1305-1314.

Kennelly, J. J. 1996. The fatty acid composition of milk fat as influenced by feeding oilseeds. Anim. Feed Sci. Technol. 60:137-152.

Knothe, G., and J. A. Kenar. 2004. Determination of fatty acid profile by ${ }^{1}$ H-NMR spectroscopy. Eur. J. Lipid Sci. Technol. 106:88-96.

Kumar, A., D. Lal, R. Seth, and V. Sharma. 2010. Detection of milk fat adulteration with admixture of foreign oils and fats using a fractionation technique and the apparent solidification time test. Int. J. Dairy Technol. 63:457-462.

Molkentin, J., and D. Precht. 1998. Comparison of gas chromatographic methods for analysis of butyric acid in milk fat and fats containing milk fat. Z. Lebensm. Unters. Forsch. A 206:213-216.

Zaïdi, F., H. Rouissi, S. Dridi, M. Kammoun, J. De Baerdemaeker, and R. Karoui. 2008. Front-face fluorescence spectroscopy as a rapid and non-destructive tool for differentiating between Sicilo-Sarde and Comisana ewe's milk during lactation period: A preliminary study. Food Bioproc. Technol. 1:143-151. 